\title{
A peculiar behaviour of liquid jet impact
}

\author{
Claudiu Pătrașcu ${ }^{1, *}$, and Corneliu Bălan ${ }^{1}$ \\ ${ }^{1}$ University Politehnica Bucharest, Hydraulics Department, Splaiul Independentei 313, Romania
}

\begin{abstract}
Coalescent masses of fluid, formed upon liquid jet impact, should exhibit either dripping or jetting regardless of the distance between the nozzles. The study reveals that above a certain flow rate, by increasing this distance, the coalescent mass enters a dripping state, when only a jetting regime is previously present. This is followed again by a jetting regime before breakup which occurs at a critical distance. The upper limit of this dripping state is achieved when Bond number is equal to unity, this result being valid below a certain critical Reynolds number.
\end{abstract}

\section{Introduction}

The stability of coalescent masses, generated by the impact of two fluid jets, is a combined problem of jet instability, ligament stretching and coalescence. Although jet instability has received a lot of attention since the early work of Rayleigh [1], numerous studies pursuing pinch-off and break-up [2], [3], some fundamental issues still remain in question, such as the role of stretching and elasticity on the subsequent dynamics. The influence of an outer medium on the dispersion relation was emphasized by Tomotika [4], which also pointed out that the stretching of a falling jet due to gravity may be the cause for a delay in break-up [5].

A fluid emerging from a nozzle does not necessarily form a jet. It can experience a dripping state or, in the case of viscoelastic fluids, even gobbling [6], understanding these phenomena having a key role in drop-on-demand technics and ink-jet printing [7], [8]. Two volumes of the same fluid, placed in contact, will merge to form one single mass of fluid, van der Waals forces or external driving conditions causing the two volumes to coalesce. This is of fundamental interest in applications such as mixing in microdevices and emulsion technology [9].

Drop coalescence in particular has been studied in relation with surface tension effects [10], induced mechanical forcing [11], finite-time singularities in mathematical modelling and topological transitions of the flow domain [12]. The volumes of fluid in question can also be fluid jets that may or may not coalesce, the transition from colliding jets to coalescent jets being a subject of material properties, angle of inclination, flow rate and jet diameter. Studies regarding coalescence of viscous cylinders by capillarity treat the subject theoretically, but a general overview of jet coalescence is still lacking. Furthermore, jet coalescence in viscous surrounding mediums can offer fundamental insight regarding the boundary that separates coalescent and non-coalescent states and new phenomena that arise with it, such as coalescence-point displacement [13].

When two capillary nozzles are placed horizontally, facing each other, and a liquid is being pumped, the liquid masses that exit the nozzles can coalesce, forming a single dripping or jetting mass of fluid. The characteristic time scales of such phenomena can be quite small when the external medium is air, but if the capillaries are submerged in a viscous liquid, the phenomenon can be slowed down. At the top end of the liquid coalescent mass a meniscus is seen connecting the two capillaries (Fig. 1). Its vertical displacement follows a periodic movement due to the periodic detachment of drops from the coalescent mass.

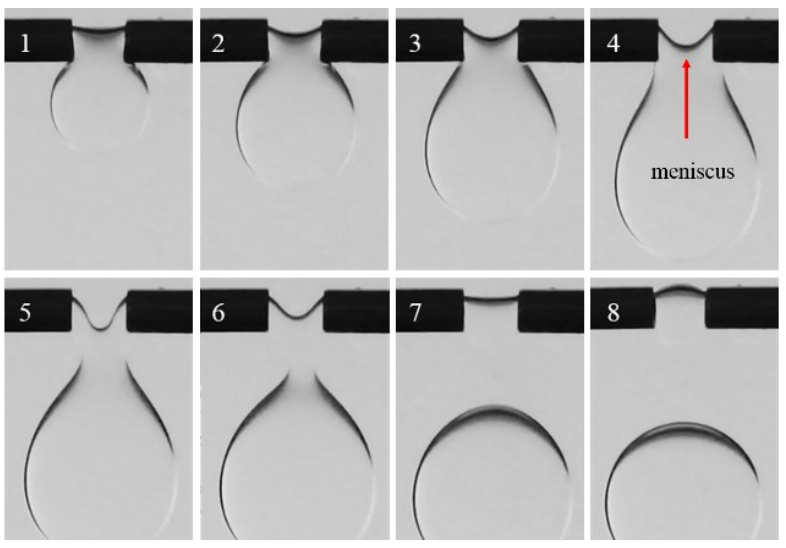

Fig. 1. Dripping mass of fluid powered by the injection from two capillaries of a solution of water and glycerin immersed in sunflower seed oil. The images show the onset of drop detachment as the bulk accumulates mass and overcomes the interfacial tension. Also, one can observe that as the weight of the bulk increases the upper meniscus that connects the capillaries deforms and increases in size. Detachment relaxes the upper meniscus decreasing its local radius of curvature. The external diameter of the nozzles, which is of $1.27 \mathrm{~mm}$, can be taken as a scale bar. (Frames are separated by $166 \mathrm{~ms}$ in the upper row and $16 \mathrm{~ms}$ in the lower row.).

* Corresponding author: claudiu.t.patrascu@gmail.com 
The study investigates the behaviour of a liquid mass of fluid, powered by the injection of two horizontally aligned capillaries, subject to an increase of the distance that separates them. The paper is structured as follows: first we provide the necessary details regarding the experimental setup, followed by the introduction of the dripping state that lies between two jetting regimes when the distance between the nozzles is changed and finally some concluding remarks.

\section{Experimental details}

Two capillaries having the same inner diameter of 0.8 $\mathrm{mm}$ where aligned horizontally by means of a mechanical mechanism. The system is submerged in sunflower seed oil, having a density of $926 \mathrm{~kg} / \mathrm{m}^{3}$ and a viscosity of $55 \mathrm{mPas}$. The injected fluids where mixtures of water and glycerine with the concentration of glycerine ranging from $33 \%$ to $75 \%$ in volume. It is worth mentioning that all mixtures are immiscible with the external medium (sunflower seed oil in this particular case). The viscosity of all fluids was measured with an Anton Paar rotational rheometer, the density with a mass per volume method and the interfacial tension using a pendant drop technique. The values of all fluids are presented in Tab. 1. As a general observation, increasing the concentration of glycerine in water produces an increase of both density and viscosity. The viscosity of the solutions relative to the concentration shows a critical value at $65 \%$, above which the viscosity increases rapidly with concentration. The interfacial tension decreases linearly with concentration.

The fluid that runs through the capillaries is being pumped at a flow rate above $10 \mathrm{ml} / \mathrm{min}$, ensuring that the two jets collide, coalesce and the coalescent mass does not break easily when the distance between the capillaries $(\delta)$ is increased. We started with the minimum distance possible and increased it by means of the mechanical mechanism. At first, we see a chaotic mixing regime taking place between the two colliding liquid jets, the result being a corrugated column of liquid that extends far downstream. By increasing the distance $\delta$ one reaches a peculiar dripping state of the coalescent mass (which will be detailed in the following section) and when reaching a critical distance, the upper meniscus, that is seen connecting the capillaries, breaks and the two jets become solitary. For concentration of glycerine above $65 \%$ the dripping state is not present, although we see some alteration of the liquid column, manifested as a diminished breakup length (Fig.2-b bottom set of images).

Tabel 1. Material properties of water-glycerin samples at $25^{\circ} \mathrm{C}$

\begin{tabular}{c|ccc} 
Sample & $\rho\left[\mathrm{kg} / \mathrm{m}^{3}\right]$ & $\eta[$ mPas $]$ & $\boldsymbol{\sigma}[\mathrm{N} / \mathrm{m}]$ \\
\hline$W+G 33 \%$ & 1098 & 3.5 & 0.023 \\
$W+G 50 \%$ & 1143 & 6.9 & 0.022 \\
$W+G 75 \%$ & 1202 & 53.5 & 0.020
\end{tabular}

\section{Dripping between jetting regimes}

The coalescent mass undergoes four states as a function of the distance between the nozzles when flow rates are above $10 \mathrm{ml} / \mathrm{min}$, as shown in Fig. 2-a. The first one is represented by an unstable regime, where the fluid emerging from the nozzles collides strongly, the coalescent mass, which ultimately falls into a fluid jet, breaking into drops by capillary instability far from the nozzles. As the distance is increased a new state is settling, which transforms the fluid jet into a dripping coalescent bulk, in this case drops being created near the nozzles (Fig. 2-b). Increasing the distance even further, the jetting regime settles again and when the critical distance is reached irreversible breakup occurs. Usually, at distances above unstable jetting, if the coalescent mass settles into a dripping or a jetting regime it will remain in that regime up until breakup.
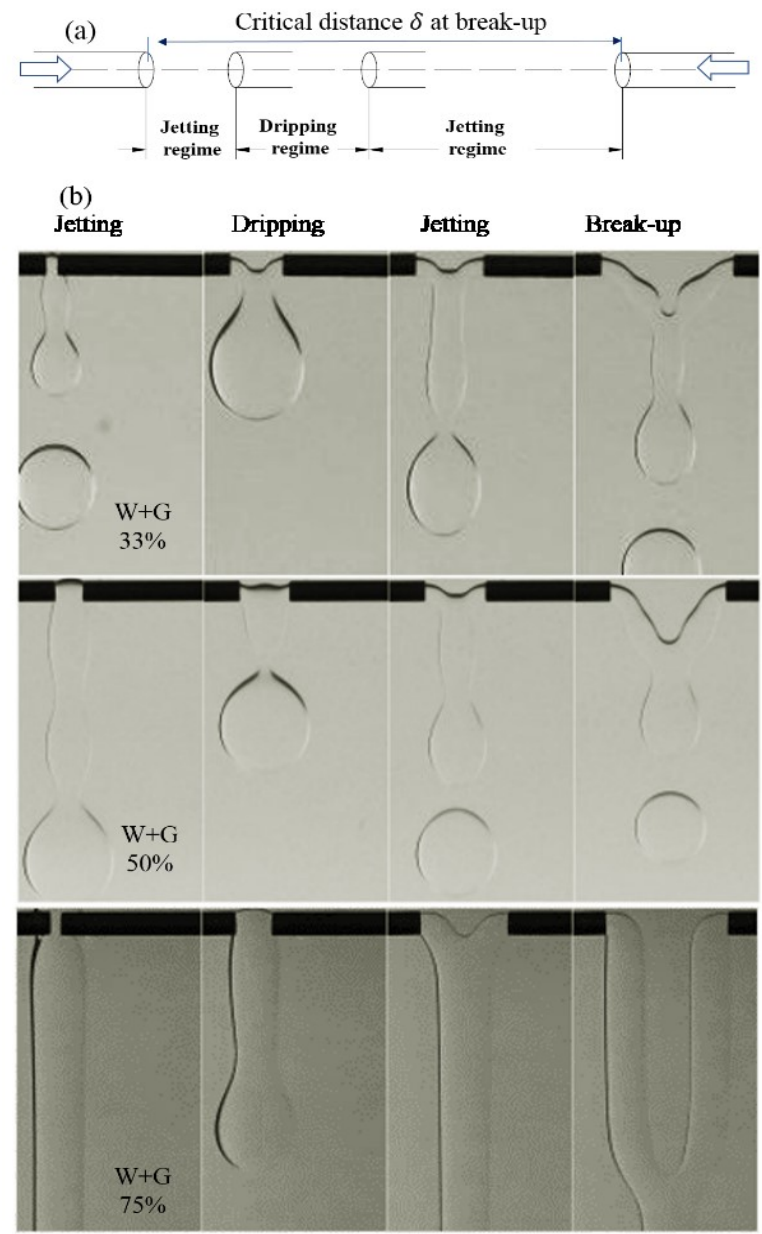

Fig. 2. a) Schematic representation of the increase of the distance between the capillaries showing four regimes: close distance jetting, dripping, jetting and breakup of the upper meniscus at the critical distance; b) Snapshots showing the effect of the increase of $\delta$ on a mass of fluid formed after the impact of two liquid jets. Three cases are presented: $33 \%$ glycerin in water $(\mathrm{W}+\mathrm{G} 33 \%)$ and $50 \%$ glycerin in water $(\mathrm{W}+\mathrm{G} 50 \%)$ which display the peculiar dripping regime (second picture from the left) and the case of $75 \%$ glycerin in water $(\mathrm{W}+\mathrm{G} 75 \%$ ) showing the diminished breakup length of a liquid jet. 
The fluid loses energy rapidly and a drop is created near the nozzles. The dripping state between jetting regimes has only a short interval of distances $\delta$ in which it appears, and Fig. 3-a shows that the upper dripping limit is reached when Bond number $\left(B o=\Delta \rho g \delta^{2} / \sigma\right)$ is equal to 1 . This represents the threshold when the reduced forces of gravity (reduced by buoyancy) are balanced by interfacial tension forces. Moreover, this behaviour is observed for water-glycerine samples with a viscosity below the critical concentration of $65 \%$, which corresponds to a Reynolds number $\left(R e=\rho V D_{0} / \eta\right)$ below 150 .

One can set from Fig. 3-b the boundary between a dripping and a jetting coalescent mass by the approximate relation:

$$
\frac{\delta}{l_{\text {Oh }}} \cong 2 R e^{2} \Rightarrow \frac{\delta}{D_{0}} \cong 2 W e,
$$

where $l_{\text {on }}$ is the intrinsic length scale of the fluid based on the Ohnesorge number, defined as: $l_{\text {oh }}=\eta^{2} / \sigma \rho$, and

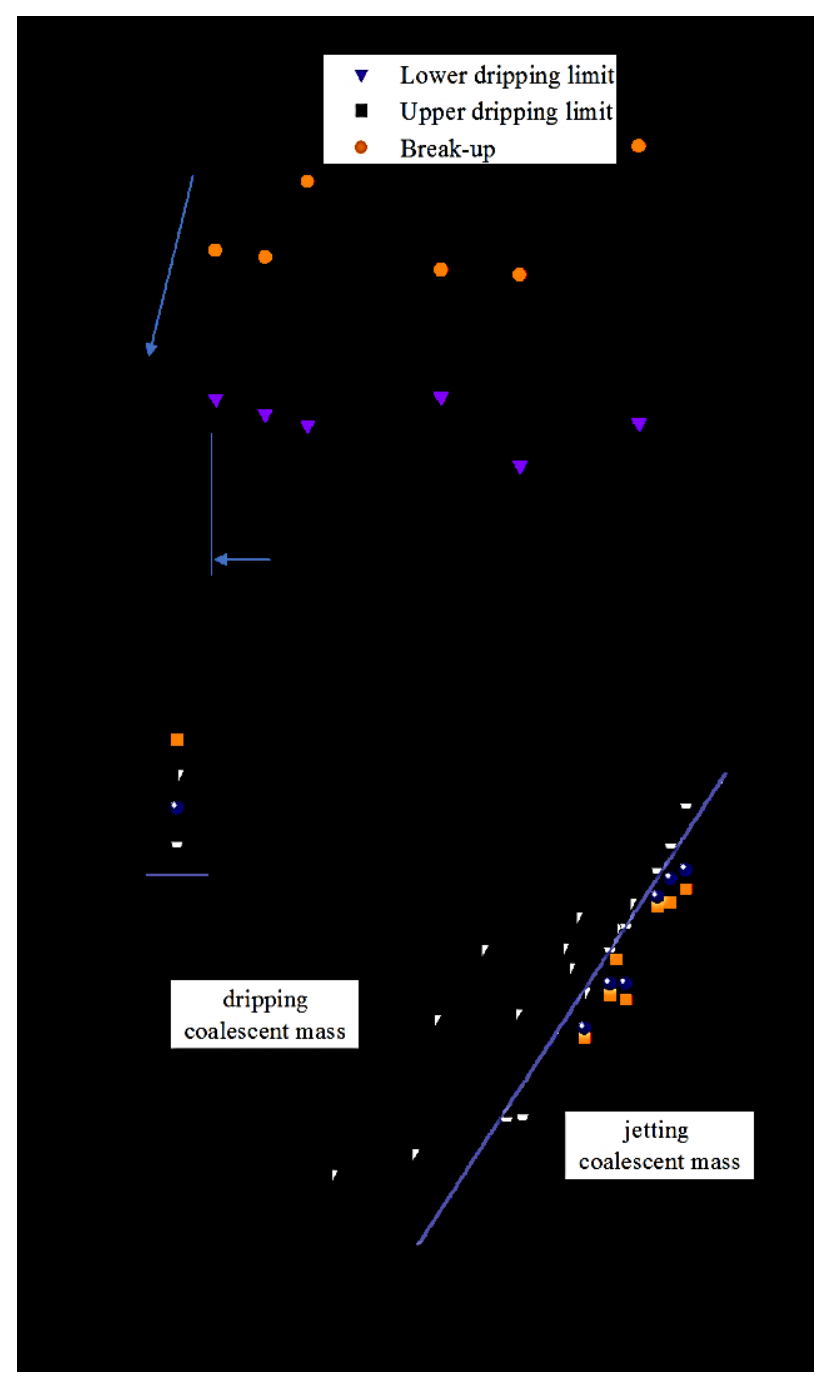

Fig. 3. a) Nondimensional representation of Bond number versus Reynolds number showing that the upper limit of the peculiar dripping regime is reached when $B \circ \approx 1$, independent of $R e$; b) The boundary between a dripping coalescent mass and a jetting coalescent mass in terms of the nondimensional distance between the capillaries and Reynolds number.

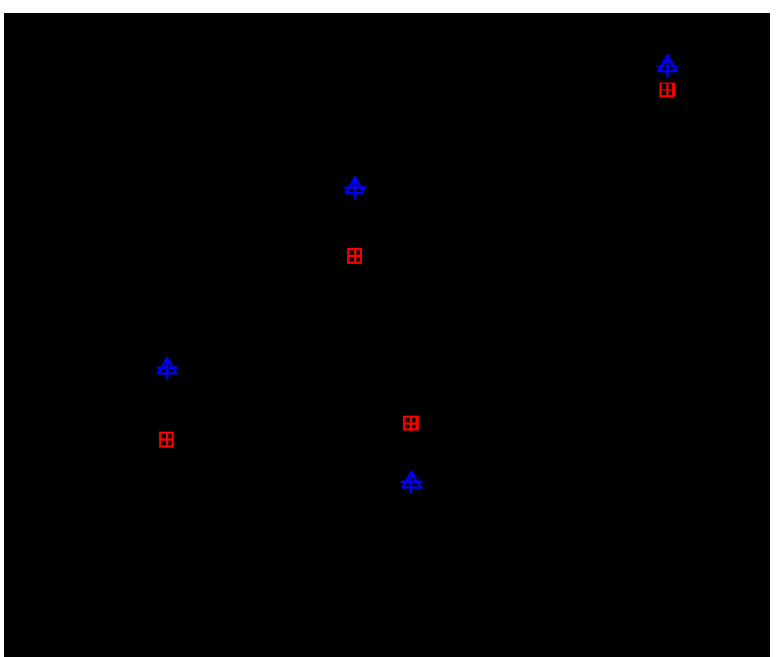

Fig. 4. Critical values for the distance between the capillaries ( $\delta)$ as function of flow rate, for three water-glycerin mixtures at which the breakup of the upper meniscus is seen. Increasing the flow rate implies an increase of critical $\delta$.

$W e$ is the Weber number defined as: $W e=\rho V^{2} D_{0} / \sigma$, with $\eta$ and $\rho$ the viscosity and the density of the injected fluid, $\sigma$ the interfacial tension, $V$ the average injection velocity and $D_{0}$ the inner diameter of the capillaries.

\section{Breakup of the upper meniscus}

When the distance between the nozzles reaches a critical value, the upper meniscus cannot sustain the weight of the bulk, therefore it breaks and the two liquid jets become independent. This is an irreversible process. We measured this critical distance for the water - glycerin samples for three different flow rates 2, 5 and $10 \mathrm{ml} / \mathrm{min}$ (Fig. 4) and found that the critical distance increases as the flow rate is increased. This is to be expected, the jets having more energy and therefore traveling larger distances. Also, we observe that for 2 and $5 \mathrm{ml} / \mathrm{min}$ the critical distance increases with concentration of glycerin, despite the fact that the capillary length (defined as $\left.l_{c}^{2}=\sigma / \Delta \rho g\right)$ decreases. This is unexpected because decreasing the capillary length would suggest lower

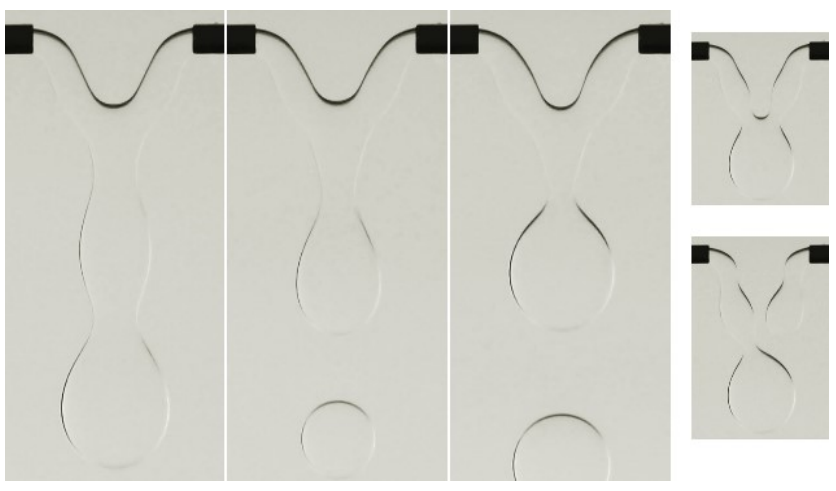

Fig. 5. Experimental observations of the breakup process for a mixture of $50 \%$ glycerin in water (frames separated by 166 $\mathrm{ms}$ ). One can observe the multiple inflection points that the interface exhibits and the asymmetrical breakup of the upper meniscus. 


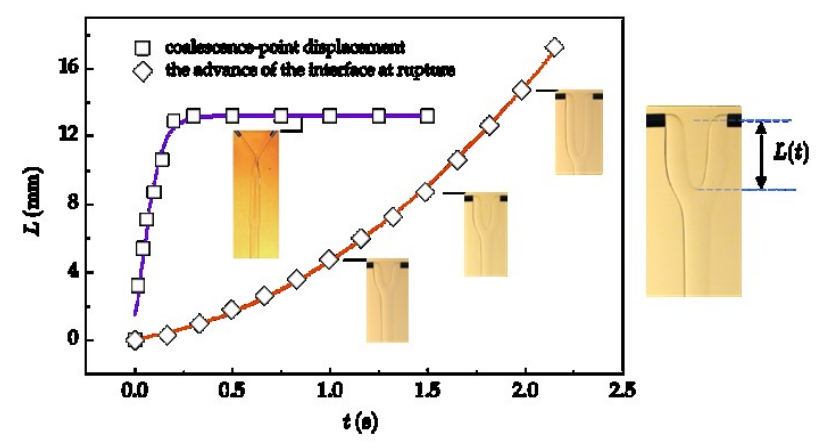

Fig. 6. Comparison between experimental values obtained for the advance of the coalescence-point displacement, in the case of the collision of two oblique liquid jets, and the advance of the interface at rupture for the specific case of two horizontally aligned nozzles.

values. If the flow rate is $10 \mathrm{ml} / \mathrm{min}$, all three values of the critical distance are quite close, but still we observe the same trend, increasing critical distance with decreasing capillary length. Fig. 5 shows the time evolution of the breakup process at this critical distance. For water-glycerin mixtures having a concentration of glycerin above $65 \%$, the dynamics of the breakup of the upper meniscus are slowed down. The upper interface starts to increase in size and eventually break, sending the two liquid jets into a state of non-collision (Fig. 6). The advance of the apex of the interface can be approximated by a second order polynomial, which suggests that, until breakup, the interface is moving with constant acceleration (Fig. 7).

Dynamic interface behavior is also encountered when two oblique liquid jets collide. The point where they collide is usually the point where thy coalesce, but for some particular choice of the material properties this point can migrate downstream [13]. Compared with the
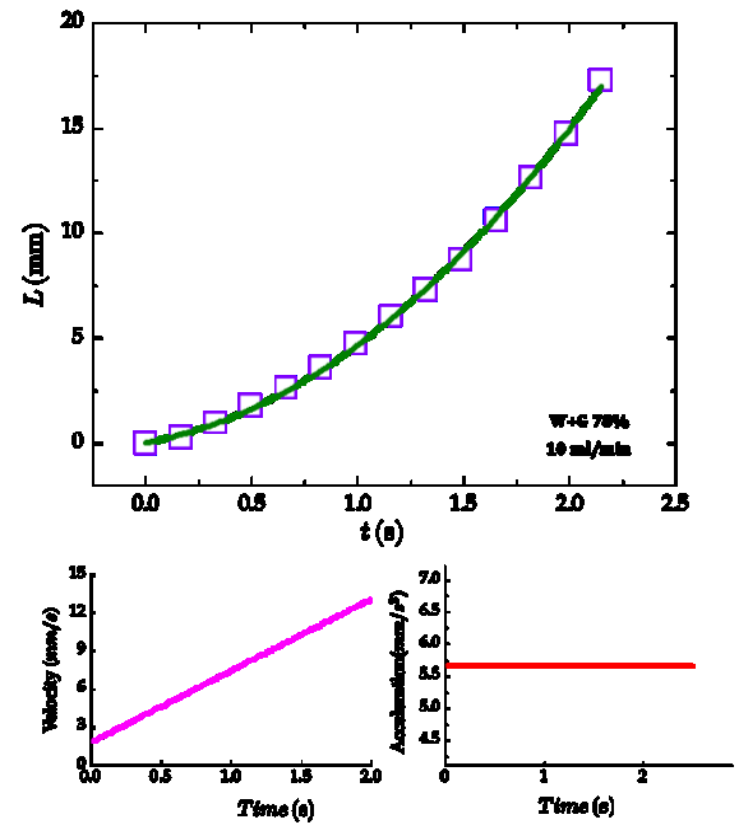

Fig. 7. Displacement velocity and acceleration of the interface at rupture for a mixture of water and $78 \%$ glycerine, injected at a flow rate of $10 \mathrm{ml} / \mathrm{min}$. The linear evolution of the velocity implies a constant advance acceleration of $5.75 \mathrm{~mm} / \mathrm{s}^{2}$. advance of the interface at rupture, the motion is decelerated.

\section{The effect of fluid elasticity}

The effect of elasticity on the coalescent mass of liquid can be inferred by using viscoelastic polyacrylamide solutions in different concentrations. The first observable effect is the appearance of a long fluid thread that connects the coalescent mass and the detached drop. The breakup time of such a thread increases with the concentration of polyacrylamide. The shape of the coalescent bulk of fluid near drop detachment is presented in Fig. 8, for three different mixtures, PAM 100,300 and $600 \mathrm{ppm}$ (parts per million). The increase of elasticity has a direct effect on the residual volume of fluid that remains attached to the nozzles, the latter increasing with elasticity. Also, the upper meniscus becomes less sensitive relative to the formation of the drop. The regime

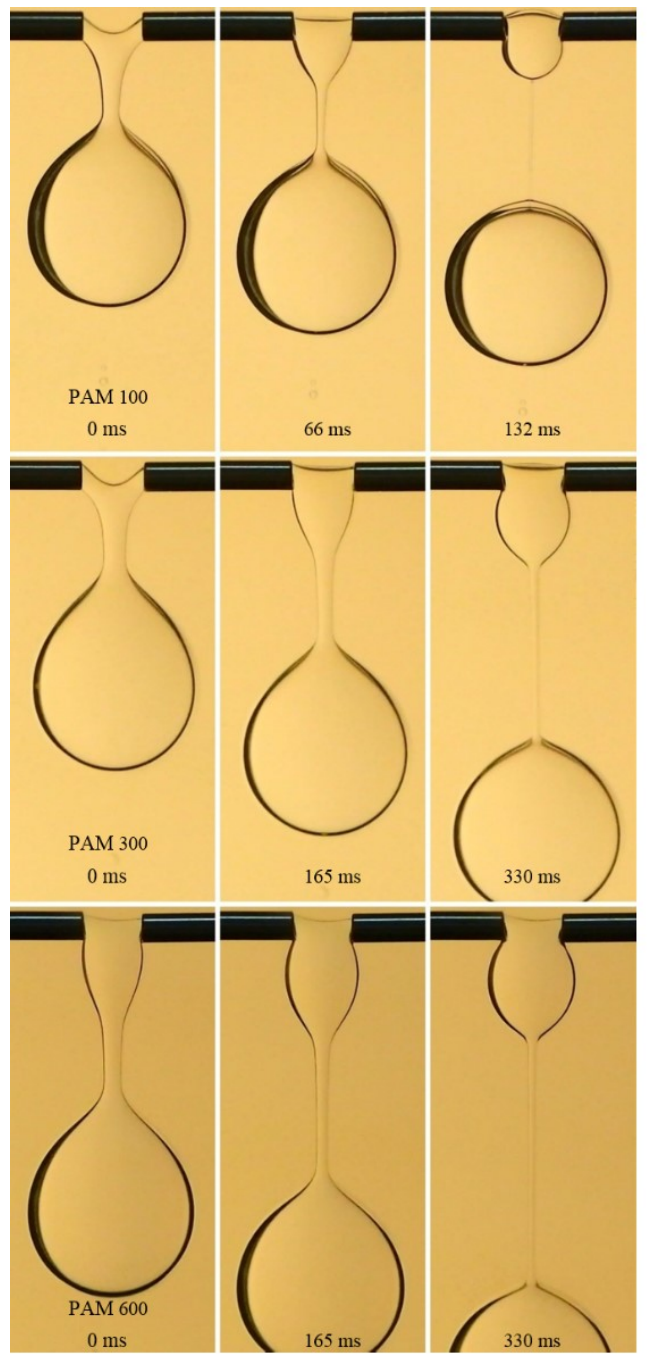

Fig. 8. The effect of increasing the elastic properties of a solution of polyacrylamide in water (PAM 100, 300 and 600 $\mathrm{ppm}$ ) on the dynamics of drop detachment, for a particular case of nozzle alignment, $\delta=2.7 \mathrm{~mm}$ distance between the nozzles and a flow rate of $Q=2 \mathrm{ml} / \mathrm{min}$. The material properties of the polyacrylamide solutions can be found in [14]. 
described in Fig. 8 is a type of absolute instability, drops forming and detaching directly from the coalescent mass near jet impact.

If, on the other hand, one increases the flow rate and keeps the distance between the nozzles fixed ( $\delta=2.7 \mathrm{~mm}$ ), the coalescent dripping mass of fluid takes the form of a jetting coalescent mass, a transition which can be seen for PAM 100 ppm in Fig. 9. Three such flow rates are depicted in Fig.9, for three viscoelastic polyacrylamide solutions. Increasing the flow rate results in an increase in volume of the liquid bridge that exits between the nozzles and a transition from a dripping regime to a jetting regime. This is most obvious for PAM 100, which is a weakly elastic polymer solution. Increasing elasticity, shifts the dripping-jetting transition towards higher values of the flow rate. The interface becomes less prone to fluctuations, this being, of course, due to the storage properties of elasticity.

The type of instability presented in Fig. 8 and 9 is an elasto-capillary type of instability which, for

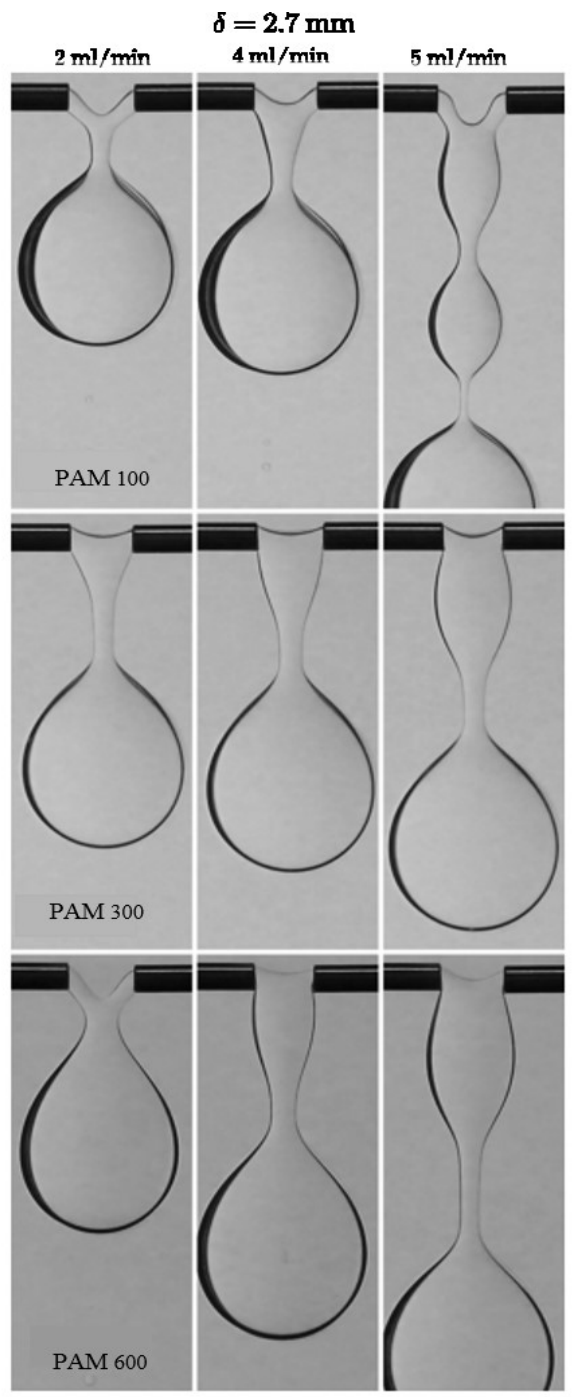

Fig. 9. The effect of increasing the flow rate on the coalescent mass of liquid for three specific viscoelastic fluids: PAM 100, 300 and $600 \mathrm{ppm}$. We observe both an increase of the residual volume that remains attached to the nozzles and a transition from dripping to jetting in the case of PAM 100.

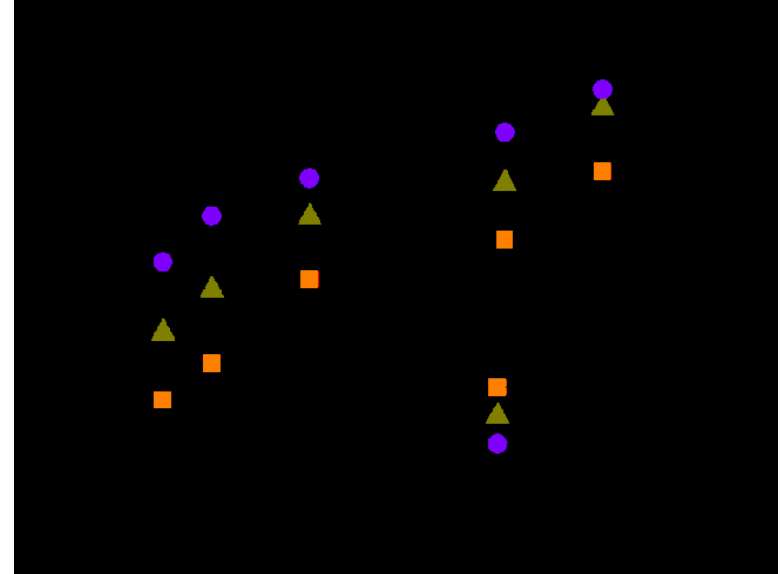

Fig. 10. Critical values for the distance between the capillaries $(\delta)$ as function of flow rate, for three polyacrylamide mixtures at which the breakup of the upper meniscus is seen. Increasing the flow rate or fluids elasticity implies an increase of critical $\delta$.

concentrated polymer solutions, remains still a challenging field of study. Compared to Newtonian mixtures the viscoelastic fluids exhibit the same trend when speaking of the critical distance at which breakup occurs, i.e. the critical distance increases with the flow rate. The addition of elasticity increases even further this distance, as it can be seen in Fig. 10, compared to the investigated Newtonian mixtures.

\section{Concluding remarks}

The paper is concerned with liquid masses of fluid formed by colliding two liquid jets, immersed in a viscous outer liquid. Such coalescent masses of Newtonian mixtures, sustained by the constant injection of fluid, can exhibit either dripping or jetting. Usually, neither regime depends on the distance that separates the nozzles, but for water-glycerine samples with concentrations below the critical value of $65 \%$ glycerine in water, by increasing the distance between the nozzles, a dripping state is observed when only jetting should be present.

This behaviour is encountered for Reynolds numbers above 30 and below 150. The upper limit that separates dripping from jetting, in this particular case, is reached when Bond number is approximately equal to 1 . Increasing the distance between the capillaries even further, the upper meniscus can rupture when a critical value is reached. This critical distance is increasing with the flow rate and decreasing with the capillary length.

At rupture, the interface advances with constant acceleration. Viscoelastic fluids in general will present higher values for the critical distance of breakup. The breakup itself is a complex phenomenon, mostly because the interface varies in time and the phenomenon is nonlinear.

We emphasize the fact that the peculiar dripping regime is not due to a misalignment of the capillaries, relative to one another, the same behaviour being encountered even if the position of the capillaries is changed with respect to the remaining two dimensions. 
This work was partially supported by the grant of the Romanian National Authority for Scientific Research, CNCS, UEFISCDI, PHANTOM - Passive flow control for heat and mass transfer enhancement of impinging jets PN-III-P4-IDPCE-2016-0758 and by the grant of the Romanian space agency ROSA, QUEST - Advanced air diffusion system of the crew quarters for the ISS and deep space habitation systems, STAR-CDI-C3-2016-577.

\section{References}

[1] L Rayleigh, Proc. London Math. Soc., vol. 10, (1878).

[2] J. Eggers, E. Villermaux, IOP Publishing, (2008)

[3] D. T. Papageorgiou, Phys. Fluids, vol. 7, no. 7, (1995)

[4] S. Tomotika, Proc. R. Soc. A Math. Phys. Eng. Sci., vol. 150, no. 870, (1935)

[5] S. Tomotika, Proc. R. Soc. A Math. Phys. Eng. Sci., vol. 153, no. 879, (1936)

[6] C. Clasen, J. Bico, V. M. Entov, G. H. McKinley, J. Fluid Mech., vol. 636 , (2009).

[7] A. U. Chen, O. A. Basaran, Phys. Fluids, vol. 14, no. 1, (2002)

[8] W. van Hoeve, S. Gekle, J. H. Snoeijer, M. Versluis, M. P. Brenner, D. Lohse, Phys. Fluids, vol. 22, no. 12 , (2010)

[9] S. T. Thoroddsen, B. Qian, T. G. Etoh, K. Takehara, Phys. Fluids, vol. 19, no. 7, (2007)

[10] M. Wu, T. Cubaud, and C. M. Ho, Phys. Fluids, vol. 16, no. 7, (2004)

[11] J. M. Frostad, A. Paul, and L. G. Leal, Phys. Rev. Fluids, vol. 1, no. 3, (2016)

[12] Y. D. Shikhmurzaev, Phys. Fluids, vol. 12, no. $10,(2000)$

[13] I. L. Omocea, C. Patrascu, M. Turcanu, C. Balan, EHB, (2016)

[14] C. Patrascu, I. L.Omocea, C. Balan, Prod. Rom. Acad., Vol. 18, no. 3 (2018) 